Key Observations for Stellar Modelling

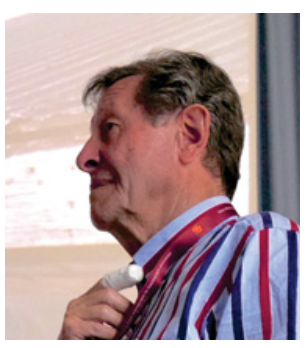

N. Grevesse (chair)

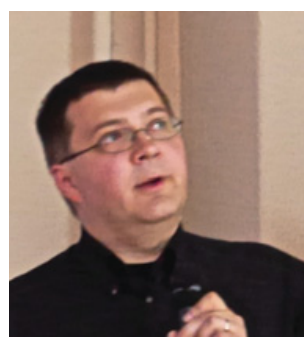

N. Przybilla

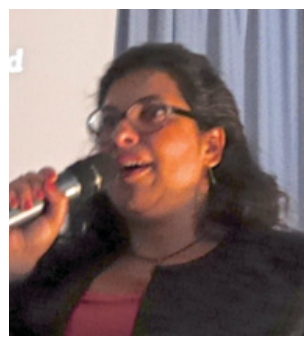

M. Chadid

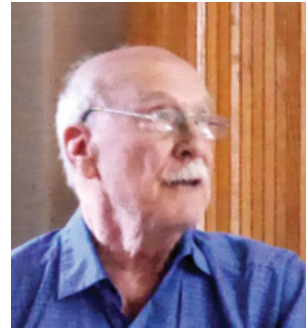

G. Preston (chair)

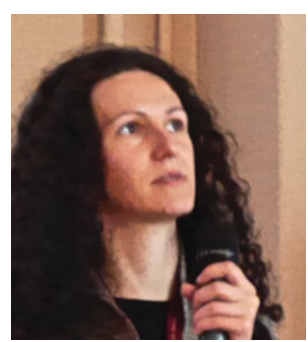

S. Bisterzo

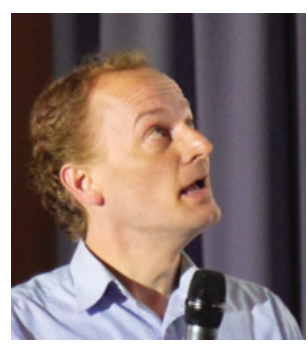

A. Korn 


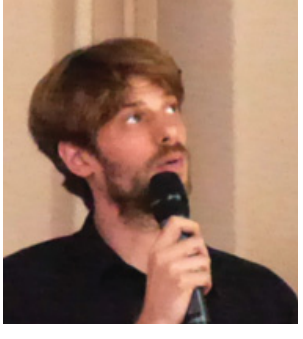

M. Castro

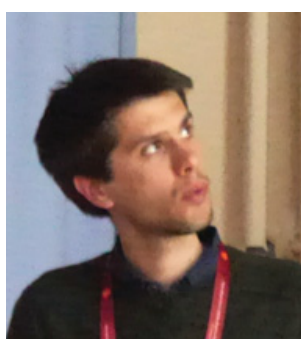

J. Silvester

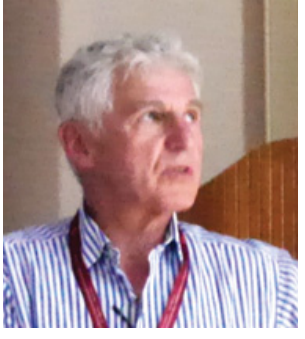

J. Landstreet

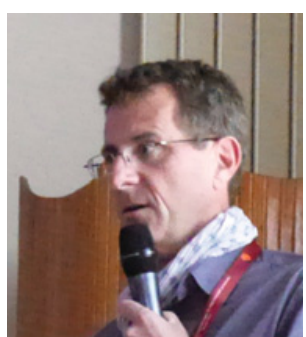

F. Thevenin 
New Advances in Stellar Physics: From Microscopic to Macroscopic Processes

G. Alecian, Y. Lebreton, O. Richard and G. Vauclair (eds)

EAS Publications Series, 63 (2013) 13-23

\title{
HOT STARS AND COSMIC ABUNDANCES
}

\author{
N. Przybilla ${ }^{1}$, M.F. Nieva ${ }^{1,2}$, A. Irrgang ${ }^{2}$ and K. Butler ${ }^{3}$
}

\begin{abstract}
Hot massive stars are ideal indicators for present-day cosmic abundances. We review results from a non-LTE study of a larger sample of early B-type stars in the solar neighbourhood by Nieva \& Przybilla (2012) and extend the analysis. Using comprehensive models with improved microphysics, novel analysis methodologies and highquality spectra it is shown that the present-day chemical composition in massive stars out to several hundred parsec distance from the Sun is highly homogeneous. Abundances for about a dozen astrophysically important chemical elements are presented, including new preliminary data on aluminium, sulphur and argon. This establishes a cosmic abundance standard, which serves as a reference facilitating chemical peculiarities in other stars to be identified. Similarities and differences to the solar standard, and implications are discussed.
\end{abstract}

\section{Introduction}

Hot massive stars are ideal indicators for present-day cosmic abundances. This is because they preserve the pristine chemical composition of their natal cloud and typically do not migrate far beyond their birth environments over their short lifetimes, in contrast to older stars. They are also unaffected by depletion onto dust grains, unlike the cold/warm interstellar medium or H II regions. From an analysis perspective, quantitative spectroscopic studies of unevolved early B-type stars are to be preferred when absolute abundances of highest accuracy and precision are desired. The reason is their simple photospheres, which are unaffected by macroscopic phenomena like strong stellar winds or convection, and microscopic atomic diffusion, which gives rise to chemical peculiarities in many other kinds of stars. However, as a slight complication, deviations from local thermodynamic

\footnotetext{
${ }^{1}$ Institute for Astro- and Particle Physics, University of Innsbruck, Technikerstr. 25/8, 6020 Innsbruck, Austria

2 Dr. Remeis Observatory \& ECAP, University of Erlangen-Nuremberg, Sternwartstr. 7, 96049 Bamberg, Germany

${ }^{3}$ University Observatory, University of Munich, Scheinerstr. 1, 81679 Munich, Germany
}

(C) EAS, EDP Sciences 2014

DOI: $10.1051 /$ eas/1363002 
equilibrium (non-LTE effects) need to be accounted for properly in line-formation calculations.

Several rather comprehensive non-LTE studies have targeted nearby early$\mathrm{B}$ main-sequence stars in the past to derive present-day cosmic abundances, e.g. Kilian (1992), Gies \& Lambert (1992), Cunha \& Lambert (1994), Morel et al. (2006). Using standard analysis techniques, these have found large uncertainties in basic stellar parameters, a tendency towards a metal-poor composition with respect to older stars like the Sun and an overall enormous range in derived elemental abundances. The latter are contrasted by a chemically homogeneous ISM gas (Sofia \& Meyer 2001) - the material the stars were formed from.

These discrepancies have recently been resolved by Nieva \& Przybilla (2012, henceforth abbreviated to NP12). Using comprehensive models with improved microphysics, novel analysis methodologies and high-quality spectra for a carefully selected star sample it was shown that the present-day chemical composition in massive stars out to several hundred parsec distance from the Sun is highly homogeneous, to the $10 \%$-level. This allowed a cosmic abundance standard to be established, which is an important complement to the only other standard abundances available, the solar values. Here we summarise the major steps in the work of NP12, present new (preliminary) results on additional chemical species, and discuss implications.

\section{Observations and analysis}

The sample of NP12 consists of bright, sharp-lined, apparently single and chemically inconspicuous stars that show no indications for the presence of circumstellar emission. An initial sample of 20 objects in associations and the field out to $\sim 400 \mathrm{pc}$ distance from the Sun was complemented by 9 additional stars in the Orion OB1 association analysed by Nieva \& Simón-Díaz (2011, NS11). The distribution of the sample stars in the solar neighbourhood is displayed in Figure 1, essentially delineating Gould's Belt.

High- $S / N$ and high-resolution echelle spectra (with $S / N>250-500$ typically, and resolving power $R=\lambda / \Delta \lambda \geq 40000$ ) were obtained with FEROS on the ESO/MPG $2.2 \mathrm{~m}$ telescope at La Silla, FOCES on the $2.2 \mathrm{~m}$ telescope on Calar Alto, FIES on the $2.5 \mathrm{~m}$ Nordic Optical Telescope on La Palma, or taken from the ELODIE (on the $1.93 \mathrm{~m}$ telescope at Observatoire de Haute-Provence) archive. All spectra have a wide wavelength coverage ( $\sim 3900-7000 \AA$, and often out to $9000 \AA)$, which is required to access all important spectroscopic indicators.

Model calculations were carried out using a hybrid non-LTE approach as discussed in detail by Nieva \& Przybilla $(2007,2008)$ and Przybilla et al. (2011). In brief, hydrostatic, plane-parallel and line-blanketed LTE model atmospheres were computed with AtLAs9 (Kurucz 1993). Non-LTE line formation computations were then performed on these model structures using updated versions of the Detall and Surface codes (Giddings 1981; Butler \& Giddings 1985). State-of-the-art non-LTE model atoms (see Table 1) relying on data from $a b$ initio computations - avoiding rough approximations wherever possible - were 


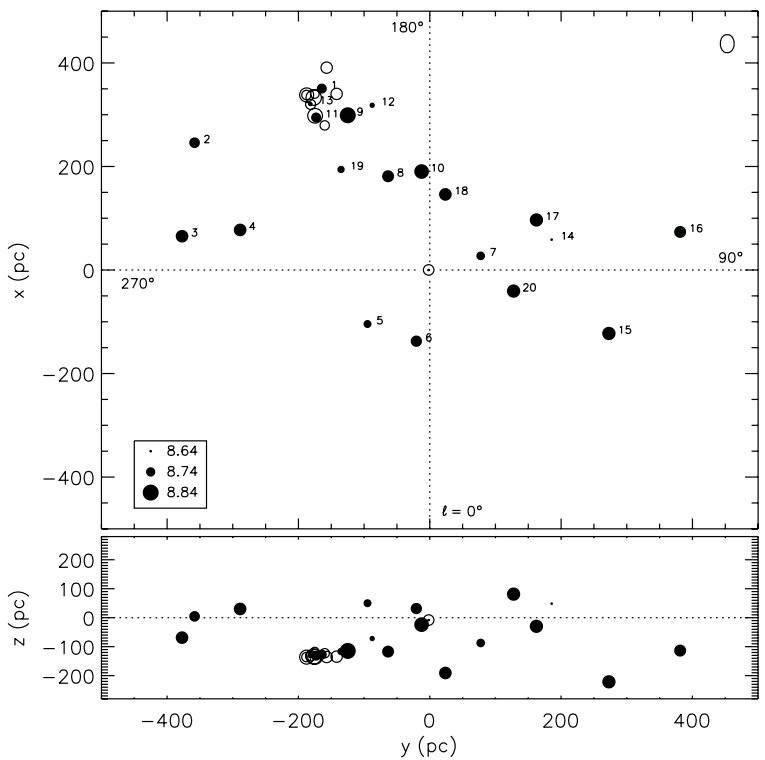

Fig. 1. Distribution of the sample stars in the solar neighbourhood. Upper panel: Galactic plane projection. Lower panel: rotational plane projection. Numbers/letters correspond to those used by Nieva \& Przybilla (2012, NP12). Open circles represent Orion stars from Nieva \& Simón-Dáz (2011). The symbol size encodes the oxygen abundance according to the figure legend. The position of the Sun is marked by $\odot$. From NP12.

Table 1. Model atoms for non-LTE calculations.

\begin{tabular}{ll}
\hline \hline Ion & Model atom \\
\hline H & Przybilla \& Butler (2004) \\
He I/II & Przybilla (2005) \\
C II-IV & Nieva \& Przybilla $(2006,2008)$ \\
N II & Przybilla \& Butler (2001), updated \\
O I/II & Przybilla et al. (2000), Becker \& Butler (1988), updated \\
Ne I/II & Morel \& Butler (2008), updated \\
Mg II & Przybilla et al. (2001) \\
Al II/III & Przybilla (in prep.) \\
Si III/IV & Becker \& Butler (1990), updated \\
S II/III & Vrancken et al. (1996), updated \\
Ar I/II & Butler (in prep.) \\
Fe II/III & Becker (1998), Morel et al. (2006), corrected \\
\hline
\end{tabular}

utilised in this step, see Przybilla (2010) for an overview of the importance of these input data.

The stellar parameter and abundance determination for early B-type main-sequence to giant stars employs an iterative analysis methodology 
(Nieva \& Przybilla 2010, NP12). Numerous spectroscopic indicators such as multiple ionization equilibria and Stark-broadened profiles of the Balmer and Paschen lines are used simultaneously to derive effective temperatures $T_{\text {eff }}$ and surface gravities $\log g$. Both, high accuracy and precision can be achieved, with $1 \sigma$ uncertainties reduced to $\sim 1-2 \%$ in $T_{\text {eff }}$ (facilitating a thorough re-calibration of

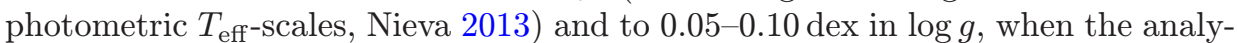
sis is performed in a self-consistent way. Absolute elemental abundances can then be constrained with unprecedented accuracy and precision: to $\sim 25 \%$ for systematic and $\sim 10-20 \%$ for random $1 \sigma$-errors. The quality of the stellar parameter determination was verified in addition by simultaneously matching the measured stellar spectral energy distributions (employing UV spectrophotometry obtained with the IUE satellite and ground-based optical and near-IR photometry) with the model fluxes and by comparing the spectroscopic with Hipparcos distances. A quality criterion for the elemental abundance determination was the absence of systematic trends of abundances with $T_{\text {eff }}$ or $\log g$. Finally, the model spectra were compared with the observed spectra, finding a good to excellent match throughout the entire analysed wavelength range, see Figure 2 for an example.

\section{A cosmic abundance standard}

The results of the abundance determinations by NP12 are visualised in Figure 3. Distribution functions for the individual elemental abundances in the star sample are displayed in comparison to literature data (see NP12 for details) and solar photospheric and protosolar abundances from Asplund et al. (2009, AGSS09). The data are normalised by the number of sample members, with the maximum value set to 1 . The bin width is chosen as the standard deviation of the individual distributions. Note that only 20 stars are considered in the histograms for $\mathrm{N}-$ the atmospheres of nine stars are mixed with $\mathrm{CN}$-processed material - as pristine abundances are of interest for constraining a Cosmic Abundance Standard (CAS).

In contrast to the earlier studies, very tight distributions are found, described by a standard deviation of typically 10\%. Mean abundances together with the standard deviations (of the sample) are given in Table 2. New preliminary data on aluminium, sulphur and argon are also given, based on 18 stars shared by the combined NS11 and NP12 sample, and a study by Irrgang et al. (in prep.). Table 2 also summarises present-day gas-phase abundances in the Orion nebula, and comparison values from different work on the solar standard (photospheric data): Grevesse \& Sauval (1998, GS98), AGSS09 and Caffau et al. (2011, CLSFB11). Solar bulk abundances, i.e. protosolar values (photospheric data corrected for the effects of diffusion), can be obtained from these by adding +0.05 dex for helium and +0.04 dex for all other elements. Technically, the fact that a larger number of B-stars is considered for the determination of the CAS - in contrast to one star defining the solar standard -, means that the uncertainties of the $C A S$-values can be expressed via the respective standard error of the mean, which amounts to 0.01 dex for all metals studied here. However, we prefer to assign the standard deviation of the sample as conservative error margins for the CAS. 


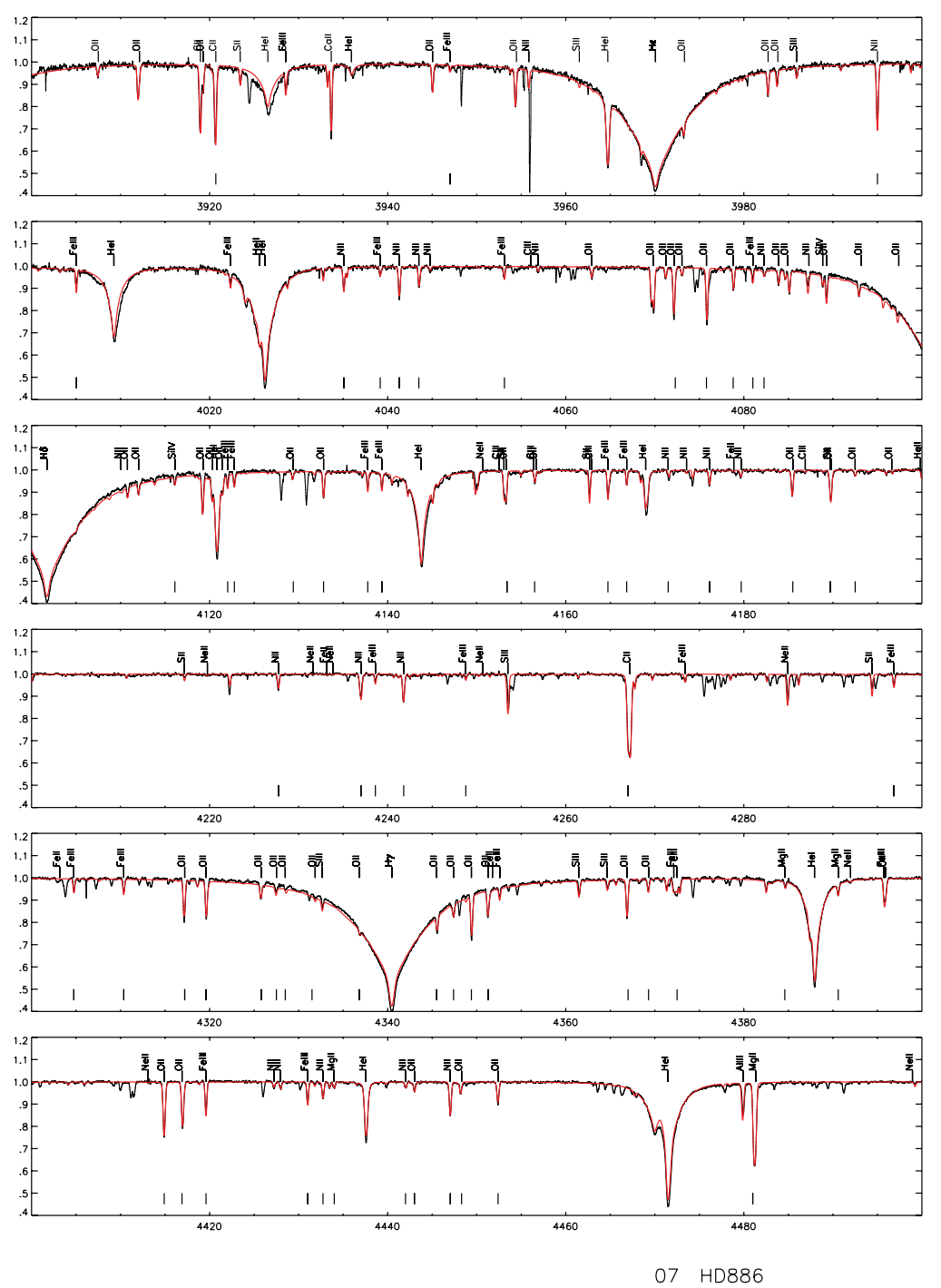

Fig. 2. Example for the comparison between the global synthetic (grey, online: red) and observed spectrum (black line) for $\gamma$ Peg (B2 IV) in the spectral range $\lambda \lambda$ 3900-4500 $\AA$. Abscissa is wavelength (in $\AA$ ), ordinate is relative flux. From NP12.

The agreement of the CAS and the Orion gas-phase abundances is excellent, except for oxygen and the refractory elements (Mg, Si, Fe), which are affected by depletion onto (silicate) dust grains. Moreover, the same degree of chemical homogeneity is recovered as for the gas-phase of the diffuse ISM out to distances of $1.5 \mathrm{kpc}$ from the Sun (Sofia \& Meyer 2001), though different absolute abundance 


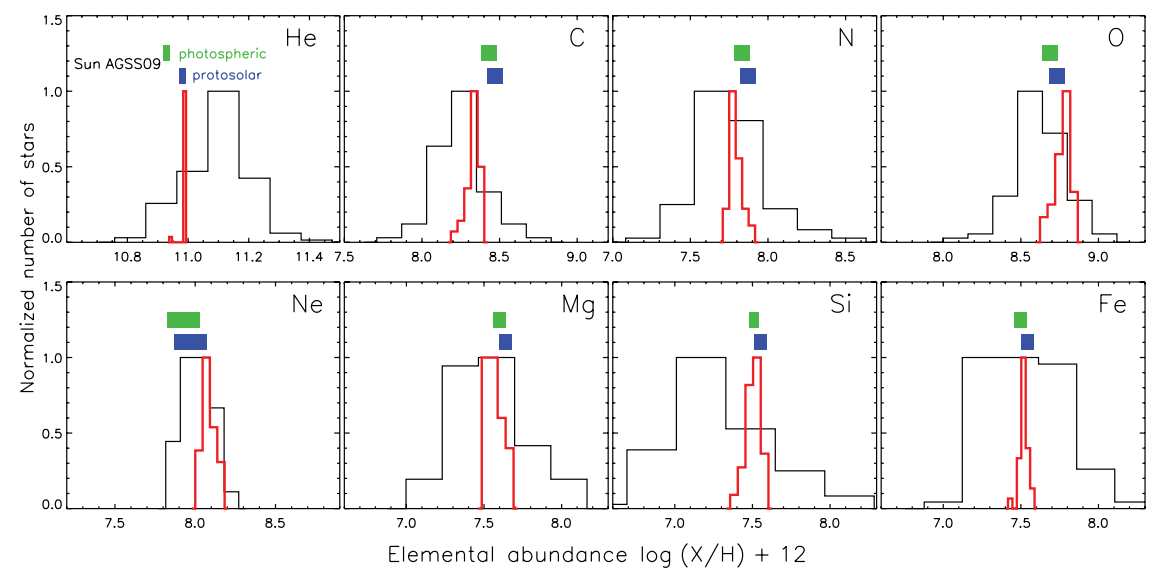

Fig. 3. Abundance distributions for the astrophysically most relevant chemical species as derived from early B-stars in the solar neighbourhood. Grey (online: red) histograms: data from NP12, establishing the cosmic abundance standard. Black histograms: literature data. Photospheric and protosolar abundances from AGSS09 are also indicated, the bars representing the range spanned by the $\pm 1 \sigma$-uncertainties. From NP12. The preliminary data on aluminium, sulphur and argon abundances show similar distributions.

Table 2. CAS abundances in comparison to the Orion nebula and the Sun ${ }^{\mathrm{a}}$.

\begin{tabular}{lccccc}
\hline \hline & Cosmic standard & Orion nebula & \multicolumn{3}{c}{ Sun $^{\mathrm{e}}$} \\
\cline { 4 - 6 } Elem. & B stars & Gas & GS98 & AGSS09 & CLSFB11 \\
\hline $\mathrm{He}$ & $10.99 \pm 0.01$ & $10.988 \pm 0.003^{\mathrm{c}}$ & & $10.93 \pm 0.01$ & \\
$\mathrm{C}$ & $8.33 \pm 0.04$ & $8.37 \pm 0.03^{\mathrm{d}}$ & $8.52 \pm 0.06$ & $8.43 \pm 0.05$ & $8.50 \pm 0.06$ \\
$\mathrm{~N}$ & $7.79 \pm 0.04$ & $7.73 \pm 0.09^{\mathrm{c}}$ & $7.92 \pm 0.06$ & $7.83 \pm 0.05$ & $7.86 \pm 0.12$ \\
$\mathrm{O}$ & $8.76 \pm 0.05$ & $8.65 \pm 0.03^{\mathrm{d}}$ & $8.83 \pm 0.06$ & $8.69 \pm 0.05$ & $8.76 \pm 0.07$ \\
$\mathrm{Ne}$ & $8.09 \pm 0.05$ & $8.05 \pm 0.03^{\mathrm{d}}$ & $8.08 \pm 0.06$ & $7.93 \pm 0.10$ & $\ldots$ \\
$\mathrm{Mg}$ & $7.56 \pm 0.05$ & $6.50:^{\mathrm{d}}$ & $7.58 \pm 0.05$ & $7.60 \pm 0.04$ & $\ldots$ \\
$\mathrm{Al}$ & $6.30 \pm 0.07^{\mathrm{b}}$ & $\ldots$ & $6.47 \pm 0.07$ & $6.45 \pm 0.03$ & $\ldots$ \\
$\mathrm{Si}$ & $7.50 \pm 0.05$ & $6.50 \pm 0.25^{\mathrm{d}}$ & $7.55 \pm 0.05$ & $7.51 \pm 0.03$ & $\ldots$ \\
$\mathrm{S}$ & $7.14 \pm 0.06^{\mathrm{b}}$ & $7.22 \pm 0.04^{\mathrm{c}}$ & $7.33 \pm 0.11$ & $7.12 \pm 0.03$ & $7.16 \pm 0.05$ \\
$\mathrm{Ar}$ & $6.50 \pm 0.08^{\mathrm{b}}$ & $6.39 \pm 0.03^{\mathrm{d}}$ & $6.40 \pm 0.06$ & $6.40 \pm 0.06$ & $\ldots$ \\
$\mathrm{Fe}$ & $7.52 \pm 0.03$ & $6.0 \pm 0.3^{\mathrm{d}}$ & $7.50 \pm 0.05$ & $7.50 \pm 0.04$ & $7.52 \pm 0.06$
\end{tabular}

a In units of $\log (\mathrm{El} / \mathrm{H})+12$, uncertainties are standard deviations; $\mathrm{b}$ preliminary values;

c Esteban et al. (2004); d Simón-Díaz \& Stasińska (2011); ${ }^{\text {e }}$ photospheric values.

values for many elements are found because of dust depletion. There is also a surprisingly good agreement of the CAS with the solar standard for many elements, though differences for some elements also exist. This is in spite of the fact that the Sun is $\sim 4.5$ Gyr older than the early B-stars (or the Orion nebula). 
Table 3. Mass fractions for hydrogen, helium and metals.

\begin{tabular}{ccrrrr}
\hline & Cosmic Standard & & \multicolumn{3}{c}{ Sun - photospheric values } \\
\cline { 2 - 2 } \cline { 5 - 6 } & B stars & & GS98 & AGSS09 & CLSFB11 \\
\hline$X$ & 0.710 & & 0.735 & 0.7381 & 0.7321 \\
$Y$ & 0.276 & & 0.248 & 0.2485 & 0.2526 \\
$Z$ & $0.014 \pm 0.002$ & & 0.017 & 0.0134 & 0.0153 \\
\hline
\end{tabular}

Finally, mass fractions for hydrogen $(X)$, helium $(Y)$ and the metals $(Z)$ resulting from the CAS are shown in Table 3. In addition to the metals investigated here - which cover the most abundant ones in the cosmos - data for all other metals up to zinc were considered for constraining $Z$, using solar meteoritic values of AGSS09, except for chlorine, where abundances from the Orion nebula were adopted (Esteban et al. 2004). Any deviations of these auxiliary data from the true cosmic values will be absorbed by the error margins of $Z$ due to their small contribution. The CAS metallicity agrees well with newly determined $Z$-values for the Sun (AGSS09, CLSFB11), despite a few differences in the chemical composition. However, it differs significantly from the long-used canonical value of $Z_{\odot}=0.02$.

\section{Implications}

In the following we wish to investigate the implications of the use of the cosmic abundance standard (instead of the solar standard) on various astrophysical fields. We concentrate on the impact of our sample data and the resulting CAS values for ISM science, for Galactic chemical evolution and the origin of the Sun and its relation to its present Galactic neighbourhood. Moreover, it provides the recommended initial chemical composition for modelling massive star evolution in the (extended) solar neighbourhood.

Chemical homogeneity: early B-type stars vs. ISM. The observational finding of a chemically homogeneous ISM out to $1.5 \mathrm{kpc}$ from the Sun (Sofia \& Meyer 2001) can be understood as a natural consequence of turbulent mixing acting on all scales. This is due to the large density variations of the gas, generated by a complex interaction of many factors such as momentum injection by stellar winds and supernova shocks, magnetic fields and self-gravity, which is supported by recent theoretical investigations. The study of NP12 provides an independent verification of these observational findings for the first time. The huge advantage of studying early-type stars is that the entire metal content can be determined using quantitative spectroscopy, with no material hidden in an observationally inaccessible reservoir like the dust-phase in the ISM.

Dust composition of the local ISM. An important open question in our understanding of the ISM is the chemical composition of the dust particles. The amount of metals incorporated into dust cannot be derived directly from observations. Only an indirect determination is feasible, by comparison of the ISM gas-phase 


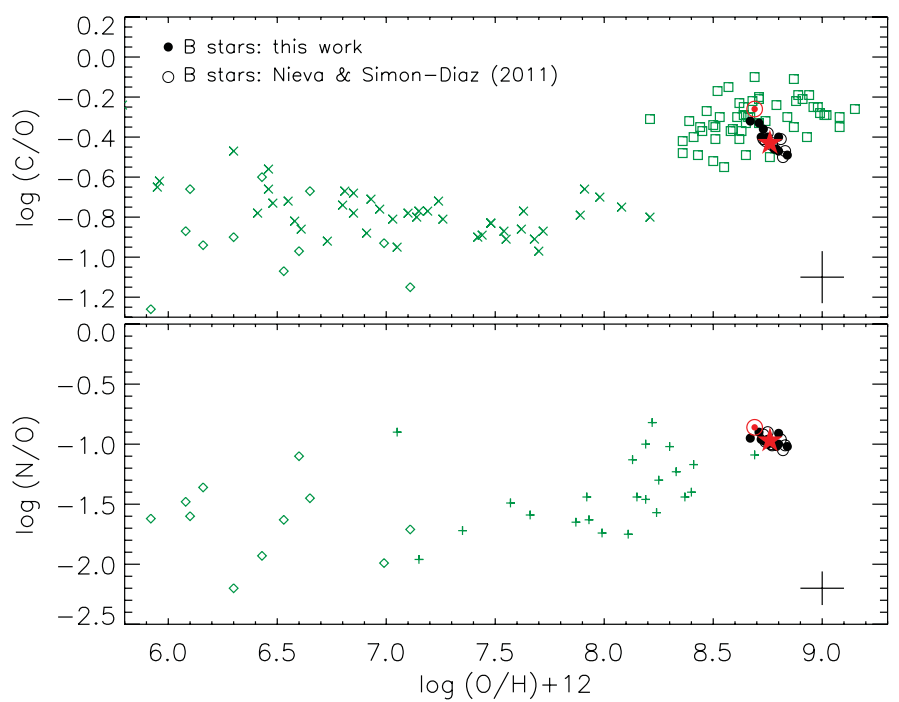

Fig. 4. Observational constraints on the Galactic chemical evolution (GCE) of CNO abundances: abundance ratios $\log (\mathrm{C} / \mathrm{O})$ and $\log (\mathrm{N} / \mathrm{O})$ vs. O abundance. Data for lowmass stars that trace the GCE over cosmic time are displayed as grey symbols (online: green), see NP12 for references to the original work. Solar abundance ratios of AGSS09 are also indicated $(\odot)$. Black dots: unmixed objects from NP12, black circles: unmixed stars from NS11, which together mark the present-day endpoint of GCE in the solar neighbourhood. The CAS is also indicated (red star). Error bars (statistical $1 \sigma$-uncertainties) typical for individual stars in NS11/NP12 are shown. From NP12.

abundances and a suitable reference unaffected by depletion onto dust grains. Using CAS values, a silicate/oxide-rich and relatively carbon-poor composition is indicated. In particular, the CAS provides sufficient oxygen to sustain the values required so that the vast majority of magnesium, silicon and iron is locked up in silicates (plus a small fraction in metal oxides) in the local diffuse ISM. About $60 \%$ of the total carbon resides in the dust phase. On the other hand, comparison of CAS values with Orion gas-phase abundances (see Table 2) implies that the H II region is devoid of carbonaceous dust. From observations of the photodissociation region in the Orion nebula it is known that polycyclic aromatic hydrocarbons disappear as the gas becomes ionized. Our results imply that photoevaporation affects all carbon-bearing dust particles in a similar manner, indicating that little carbon is incorporated initially in graphite (the most stable form of carbon under interstellar conditions) in the ISM. There is only weak evidence for the destruction of silicate grains in the Orion nebula.

Galactochemical evolution: present-day abundances. Nucleosynthesis in successive generations of stars has enriched the cosmic matter with heavy elements ever since the first Population III stars were born. Studies of long-living objects like solar-type stars allow the cosmic enrichment history to be traced and the specific 


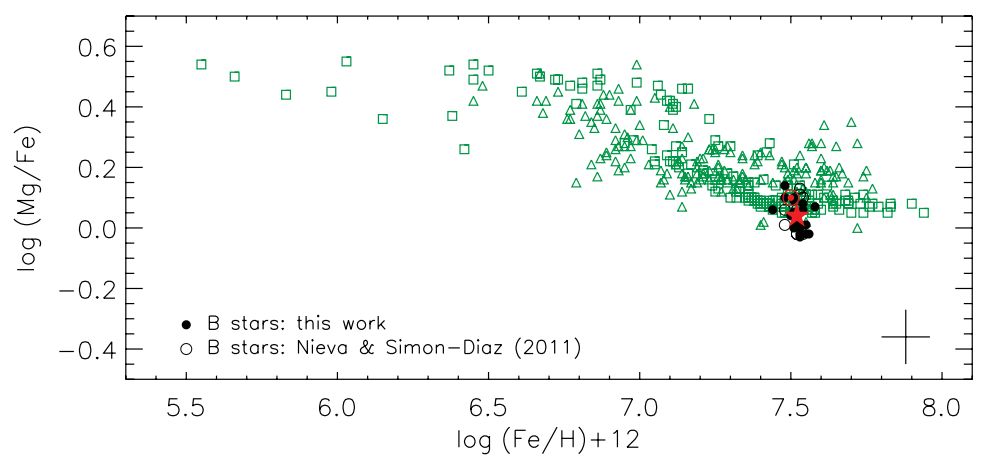

Fig. 5. Evolution of Galactic $\alpha$-process vs. iron-group elements: $\log (\mathrm{Mg} / \mathrm{Fe})$ as a function of Fe abundance. Literature data on solar-type stars are displayed as grey symbols (green online) - squares: Fuhrmann (1998, 2004), triangles: Edvardsson et al. (1993). All other symbols as in Figure 3. From NP12.

production sites of individual elements to be constrained. The CAS provides reliable present-day reference points for anchoring galactic chemical evolution (GCE) models to observation, as it marks the present-day endpoint of galactochemical evolution, in particular for the Milky Way. Observational constraints on the evolution of Galactic $\mathrm{C} / \mathrm{O}$ and N/O abundance ratios are displayed in Figure 4, and of $\mathrm{Mg} / \mathrm{Fe}$ ratios in Figure 5. GCE models not only have to reproduce the behaviour of the observation but also have to match the present-day composition as a boundary condition. It is therefore important for the entire modelling which reference values are used, solar or CAS abundances. In particular the differences in the $\mathrm{C} / \mathrm{O}$ ratio are appreciable, also with respect to the majority of nearby solar-type stars, amounting to almost $50 \%$. Taken at face value this difference indicates that the $\mathrm{C} / \mathrm{O}$ enrichment of the ISM in the present-day solar neighbourhood occurred more slowly than at the Sun's place of birth. Note that the Sun may be viewed as an extreme but still compatible case in terms of the distribution of the stars from the present sample in the $\mathrm{O}-\mathrm{C} / \mathrm{O}$ diagram, but the absolute values for the carbon abundance differ significantly. On the other hand, the solar and CAS data on N/O are compatible. The occurrence of $\alpha$-enhancement in the old stars of the halo and of the thick disk, Figure 5, is well understood in terms of the different evolution timescales of supernovae of type Ia and II. The CAS Mg/Fe-ratio is compatible with the data from the Sun and from nearby solar-type stars, though it is somewhat low.

The Sun: an immigrant to its current neighbourhood. The similarities and differences between the CAS and the solar standard imply that the Sun and also other metal-rich stars in the solar neighbourhood have not been born in a location close to the solar circle, but have migrated outwards from the inner Galactic disk over their lifetime. Birthplaces can be estimated by accounting for the enrichment of the ISM by GCE and the presence of radial abundance gradients in the 
Galaxy (see NP12 for details). The telltale signature of the Sun's origin comes mainly from the difference between the solar and the CAS C/O ratio. This indicates a birthplace at a distance around 5-6 kpc from the Galactic Centre for the Sun. However, tracing its exact orbit back is a highly complex task, as passages near other stars or molecular clouds, and dynamical interactions with spiral arms prevent a straightforward integration backward in time.

MFN acknowledges a FFL stipend from the University of Erlangen-Nuremberg and AI support by a research scholarship from the Elite Network of Bavaria, and from the German Research Foundation (DFG) through grant He 1356/45-2.

\section{References}

Asplund, M., Grevesse, N., Sauval, A.J., \& Scott, P., 2009, ARA\&A, 47, 481 (AGSS09)

Becker, S.R., 1998, ASP Conf. Ser., 131, 137

Becker, S.R., \& Butler, K., 1988, A\&A, 201, 232

Becker, S.R., \& Butler, K., 1990, A\&A, 235, 326

Butler, K., \& Giddings, J.R., 1985, in Newsletter of Analysis of Astronomical Spectra, No. 9 (Univ. London)

Caffau, E., Ludwig, H.-G., Steffen, M., et al., 2011, Sol. Phys., 268, 255 (CLSFB11)

Cunha, K., \& Lambert, D.L., 1994, ApJ, 426, 170

Edvardsson, B., Andersen, J., Gustafsson, B., et al., 1993, A\&A, 275, 101

Esteban, C., Peimbert, M., García-Rojas, J., et al., 2004, MNRAS, 355, 229

Fuhrmann, K., 1998, A\&A, 338, 161

Fuhrmann, K., 2004, Astron. Nachr., 325, 3

Giddings, J.R., 1981, Ph.D. Thesis (Univ. London)

Gies, D.R., \& Lambert, D.L., 1992, ApJ, 387, 673

Grevesse, N., \& Sauval, A.J., 1998, Space Sci. Rev., 85, 161 (GS98)

Kilian, J., 1992, A\&A, 262, 17

Kurucz, R.L., 1993, CD-ROM No. 13 (SAO, Cambridge, Mass.)

Morel, T., \& Butler, K., 2008, A\&A, 487, 307

Morel, T., et al., 2006, A\&A, 457, 651

Nieva, M.F., 2013, A\&A, 550, A26

Nieva, M.F., \& Przybilla, N., 2006, ApJ, 639, L39

Nieva, M.F., \& Przybilla, N., 2007, A\&A, 467, 295

Nieva, M.F., \& Przybilla, N., 2008, A\&A, 481, 199

Nieva, M.F., \& Przybilla, N., 2010, EAS Publications Series, 43, 167

Nieva, M.F., \& Przybilla, N., 2012, A\&A, 539, A143 (NP12)

Nieva, M.F., \& Simón-Díaz, S., 2011, A\&A, 532, A2 (NS11)

Przybilla, N., 2005, A\&A, 443, 293

Przybilla, N., 2010, EAS Publications Series, 43, 115

Przybilla, N., \& Butler, K., 2001, A\&A, 379, 955

Przybilla, N., \& Butler, K., 2004, ApJ, 609, 1181 
Przybilla, N., Butler, K., Becker, S.R., et al., 2000, A\&A, 359, 1085

Przybilla, N., Butler, K., \& Kudritzki, R.P., 2001, A\&A, 369, 1009

Przybilla, N., Nieva, M.F., \& Butler, K., 2011, J. Phys.: Conf. Ser., 328, 012015

Simón-Díaz, S., \& Stasińska, G., 2011, A\&A, 526, 48

Sofia, U.J., \& Meyer, D.M., 2001, ApJ, 554, L221

Vrancken, M., Butler, K., \& Becker, S.R., 1996, A\&A, 311, 66 